\title{
IMPROVING GROWTH AND YIELD OF COWPEA PLANT BY FOLIAR APPLICATION OF CHITOSAN UNDER WATER STRESS \\ Farouk, S. ${ }^{1}$ and Amany R. Abd EL Mohsen ${ }^{2}$ \\ 1. Agric. Botany Dept., Fac. Agic., Mans. University, Egypt. \\ 2. Botany Department, National Research Centre, Dokki, Giza, Egypt
}

\begin{abstract}
Water stress impaired cowpea plant growth and decreased ion percentage and chlorophyll and carbohydrate concentration in the shoot as well as yield and its quality. Foliar-applied chitosan, in particular $250 \mathrm{mg} / \mathrm{l}$, increased plant growth, yield and its quality as well as physiological constituents in plant shoot under stressed or nonstressed conditions as compared to untreated plants.

Anatomically, water stress decreased thickness of leaf blade at midrib region, thickness of mesophyll tissue, thickness of midrib vascular bundle. Treatment with chitosan, in particular, $250 \mathrm{mg} / \mathrm{l}$ and their interactions with stress conditions increased all the above mentioned parameters in either non-stressed or stressed plants. It is suggested that the severity of cowpea plants damaged from water stress was reduced by $250 \mathrm{mg} / \mathrm{l}$ chitosan application.

Keywords: water stress, chitosan, cowpea, anatomy, growth, yield
\end{abstract}

\section{INTRODUCTION}

World population was increased at an alarming rate and it was expected to reach about six billion by the end of year 2050. On the other hand, food productivity is decreasing due to the effect of various abiotic stresses and climatic changes; therefore minimizing these losses is a major area of concern for all nations to cope with the increasing food requirements. In the face of a global scarcity of water resources, drought has already become a primary factor in limiting crop production in the world. At present, around $18 \%$ of the global farmland is irrigated (more than 240 million hectares) produced about $40 \%$ of the global food supply (Somerville and Briscoe 2001). Permanent or temporary water deficit stress limits the growth and distribution of natural and artificial vegetation and the performance of the cultivated plants more than any other environmental factor (Shao et al. 2009). In Egypt, water availability is considered the prime constraint that determines the addition of new cultivated areas. Agricultural expansion needs a huge amount of available irrigation water which is already not sufficient to meet al the expected demands.

The responses of plants to drought vary greatly depending on species and stress severity (Mullet and Whitsitt 1996). Higher plants respond to water deficit in several ways, stomatal closure, leaf rolling, and osmotic adjustments and reduction and consequently a decrease in cellular expansion and alteration of various essential physiological and biochemical processes that can affect growth and productivity and yield quality (Costa et al. 2008, Lobato et al. 2008, Hefny 2011). In this concern, Carvalho et al. (2004) found that, lupines cultivars tended to accumulate crude protein and 
carbon compounds in the seeds at the end of the water stress period (15 days after anthesis). However, Jansen (2008) recorded insignificant effect of water stress on protein content when imposed at the same stage.

The sustainable management of water resources is a priority for agriculture also for the temperate regions, e.g. the Mediterranean basin, where dry and hot summer usually occurs, and drought events may have a large impact on both productivity and crop quality. In this context, Bittelli et al. (2001) reported that occasional or episodic drought events may be counteracted through the use of antitranspirants. These compounds are applied to foliage to limit the water loss. They include both film-forming and stomata closing compounds, able to increase the leaf resistance to water vapor loss thus improving plant water use to assimilate carbon, and, in turn, the production of biomass or yield (Tambussi and Bort 2007). Another approach to reduce water loss due to transpiration is by increasing the reflection of sunlight from leaves, through reflectant type of antitranspirants, thus limiting the water loss deputed to evaporative leaf cooling (Gaballah and Moursy 2004).

Among antitranspirant compounds, chitosan (CHT) has previously proved to be effective in pepper (Bittelli et al. 2001). CHT is a natural, low toxic and low expensive compound biodegradable, and environmentally friendly with various applications in agriculture, obtained by deacetylation of chitin. In agriculture, $\mathrm{CHI}$ has been used in seed, leaf, fruit and vegetable coating, as fertilizer and in controlled agrochemical release, to increase plant product (New et al. 2004), to protect plants against microorganisms (Farouk et al. 2008), to protect plants against oxidative stress (Guan et al. 2009) and to stimulate plant growth (Farouk et al. 2008, 2011). In the latter studies, a positive effect of $\mathrm{CHI}$ was observed on the growth of roots, shoots and leaves of various plant species. Similar results were determined within sweet pepper and radish (Ghoname et al. 2010, Farouk et al. 2011). In addition, foliar applications with $\mathrm{CHI}$ resulted in higher vegetative growth and improvement fruit quality of cucumber (Farouk et al. 2008). For other cultivated plants, Bittelli et al. (2001) reported that foliar application of $\mathrm{CHI}$ decreased transpiration in pepper plants, and reduced water use by $26-43 \%$ while maintaining biomass production and yield. Abdel-Mawgoud et al. (2010) on strawberry showed that $\mathrm{CHI}$ application improved plant height, number of leaves, fresh and dry weights of the leaves and yield components. Fruit quality in terms of average weight of individual fruits and TSS showed similar trends. Recently, Sheikha and AL-Malki (2011) indicate that application of the Chitosan's different concentrations enhancing bean shoot and root length, fresh and dry weights of shoots, roots and leaves area as well as the level of chlorophyll in leaves. The mechanisms of $\mathrm{CHI}$ on counteracting the harmful effect of water stress are not well understood and there are a few reports on this concern. Transcriptional activation, induced by both $\mathrm{CHI}$ and jasmonic acid, of genes encoding phenylalanine ammonia lyase and protease inhibitors, suggests that $\mathrm{CHI}$ may influence pathways involving jasmonic acid (Doares et al. 1995). Jasmonates exhibit some activities similar to the plant hormone abscisic acid (ABA), which plays a key role in the regulation of water use by plants. Increased levels of ABA result in closure of stomata and 
reduced transpiration (Leung and Giraudat 1998). These authors demonstrated that $\mathrm{CHI}$ inhibited light-induced opening of stomata in tomato and Commelina communis via inducing $\mathrm{H}_{2} \mathrm{O}_{2}$ production in the guard cells. The reported effects of $\mathrm{CHI}$ on stomatal aperture suggest the possibility that $\mathrm{CHI}$ might be a valuable antitranspirant with useful agricultural applications.

Cowpea (Vigna unguiculata (L) Walp) is one of the ancient grain legumes valued for its nutritional value, especially high protein content $(25 \%)$, flavor and short cooking time (Ogbonnaya et al. 2003). The crop also has ability to maintain soil fertility through its excellent capacity to fix atmospheric nitrogen and thus does not require very fertile land for growth (Lobato et al. 2006). Moreover, cowpea forms an integral part of a sustainable agriculture and land use system (Ogbonnaya et al. 2003). The total cultivated area of this crop in Egypt was estimated by about 9155 feddan for dry seed production in the year of 2008 with a mean production of $980 \mathrm{~kg} / \mathrm{feddan}$. Also, the estimated area for fresh pods was $10064 \mathrm{~kg} / \mathrm{feddan}$ with a mean production of 5.19 ton/feddan (Dept. Agric. Statistics, ministry of Agriculture, Giza, Egypt, 2008).

The improvement in water economy may probably help water stressed plants in maintaining their physiological and biochemical processes, at least, at an acceptable base line. To the best of our knowledge there has also been no previous report regarding the effects of foliar applied $\mathrm{CHI}$ on cowpea plant growth and yield. Therefore, the objective of this work was to explore the possible role of $\mathrm{CHI}$ on improving drought tolerance in cowpea plants.

\section{MATERIALS AND METHODS}

Two pot experiments were conducted in the experimental farm and laboratory of Agricultural Botany Department, Faculty of Agriculture, Mansoura University, Egypt during the two successive seasons of 2007 and 2008. Cowpea seed "Vigna unguiculata (L.) Walp. cv Cream 7" was obtained from the legume Research Institute, Agricultural Research Centre, Ministry of Agriculture, Giza, Egypt. The seeds were sterilized with 1.5\% chlorox, washed three times with distilled water, and then coated with $\mathrm{N}$-fixer okadeen (Rhizobia) that was obtained from General Organization for Agriculture Equalization Fund (GOAFE), Ministry of Agriculture. Egypt.

Sowing was took place on $15^{\text {th }}$ and $10^{\text {th }}$ April in both seasons respectively. The pots were arranged in a complete randomized block design with three replications. Plastic pots $(50 \mathrm{~cm}$ inner diameter and $30 \mathrm{~cm}$ in length) filled with $25 \mathrm{~kg}$ air dried soil were used. The soil characteristics were as follows: sandy loam in texture, sand, $80 \%$; silt, $15.5 \%$; clay, $4.5 \%$; $\mathrm{pH}, 7.8$; $\mathrm{EC}, 0.4 \mathrm{dSm}^{-1}$ and organic matter $0.45 \%$. After sowing, irrigation was applied to supply seedlings with $100 \%$ available water, at two days intervals till the seedlings reached the fourth leaf stage. The seedlings were thinned to leave seven plants per pot. Phosphorous and potassium fertilizers were added to the soil before sowing at the rate of $5 \mathrm{~g} \mathrm{P}_{2} \mathrm{O}_{5}$ in the form of calcium super phosphate $\left(15.5 \% \mathrm{P}_{2} \mathrm{O}_{5}\right)$ and $2 \mathrm{~g} \mathrm{~K}_{2} \mathrm{O}$ in the form of potassium sulphate 
(48\%). Ammonium nitrate (33.5\%) was added at the rate of $4 \mathrm{~g} \mathrm{~N} /$ pot in two equal portions; the first during the seedling stage and the second at the beginning of flowering time. After that the pots were divided into three groups for water stress treatments, each group was divided into four subgroups for chitosan foliar application. The soil moisture for all pots was kept at $80 \%$ field capacity "FC" until 15 days after sowing (DAS). After that, the water stress treatments were initiated. Pots were subjected to one of the three water stress treatments; a well watered control, $80 \% \mathrm{FC}$ and two water stress treatments; moderate at $50 \% \mathrm{FC}$ and severe $30 \% \mathrm{FC}$ water stress. In the stressed treatments, moisture levels were allowed to fall from the initial $80 \%$ FC to $50 \%$ FC and $30 \%$ FC, respectively. All pots were weighed every two days. The loss in pots weight represents transpiration and evaporation. Cumulative water loss was added to each pot to compensate transpiration and evaporation. Accumulated water loss was calculated as the differences in pots weights between successive weights. At 40,50 and 60 days from sowing, the plants were sprayed with either tap water or chitosan at 125, 250 or $500 \mathrm{mg} / \mathrm{l}$ till dripping using small pressure pump after adding tween 20 as a wetting agent at concentration of $0.5 \%$.

\section{Data Recorded:}

Three uniform plants were uprooted from each pot at the full blooming stage ( 80 days from sowing) to measure certain morphological and physiological characteristics as well as leaflet anatomy

Morphological characteristics:

The plants were cleaned and the following parameters were determined: plant height, number of leaves/plant and number of branches/plant. Shoot fresh and dry weight/plant were estimated by drying plant at $70^{\circ} \mathrm{C}$ until constant weight.

\section{Chemical Composition of Leaves:}

1. Total chlorophyll was extracted for $24 \mathrm{hr}$ at room temperature in methanol after adding traces of sodium carbonate and determined spectrophotometrically (Spekol 11, Uk) according to Lichtenthaler and Wellburn (1985).

2. Total carbohydrates concentration was estimated using the anthrone method as described by Sadasivam and Manickam (1996).

3. Mineral constituents: Dry shoot $(0.2 \mathrm{~g})$ was digested using $5 \mathrm{~cm}$ from the mixture of sulfuric and perchloric acid $\left(\mathrm{HClO}_{3} / \mathrm{H}_{2} \mathrm{SO}_{4}\right.$ 1:1 v:v) until a sample had become clear, cooled and made up to $50 \mathrm{ml}$ using deionized water. Total nitrogen was determined by micro-kjeldahl method. Potassium was determined by flame-photometerically (Kalra 1998). Phosphorous was also estimated by using ammonium molybdate and ascorbic acid (Cooper 1977).

Leaflet structure: Small pieces from the midrib region of the $3^{\text {rd }}$ upper leaflet (second season) were fixed in formalin aceto alcohol for $48 \mathrm{~h}$, then dehydrated via $\mathrm{n}$-butanol series and embedded in paraffin wax $\left(52-54{ }^{\circ} \mathrm{C}\right.$ melting point). Sections were prepared using a rotary microtome at 15-17 $\mu \mathrm{m}$ thickness and stained with safranin/light green and finally mounted in canada balsam. Selected sections were examined using light microscope to determine the anatomical changes in leaflets. 
Total Yield and its quality:

At harvest time (140 days from sowing) the total yield per plant was recorded. Seed quality represented by their concentrations of nitrogen, phosphorous, potassium, protein and carbohydrates were determined in the dry seeds as previously described in shoots. Finally, the protein percentage in dry seeds was accounted by multiplying nitrogen content by 6.25 .

Statistical analysis:

All data were analyzed statistically using One-way ANOVA to follow by Duncan's Multiple Range Test (DMRT) by COSTAT software. The values are mean $\pm S D$ for three samples in each group. $P$ values $<0.05$ were considered as significant.

\section{RESULTS}

\section{Growth parameters}

Generally, severe reduction in plant growth, manifested by smaller, chlorotic, wilted, and rolled leaves was recorded due to water stress. From the results presented in Table (1), it could be concluded that plant growth characters i.e. plant height, branches and leaf number per plant, and shoot fresh and dry weight were significantly decreased due to water stress in both growing seasons.

Table (1): Effect of water stress and chitosan and their interactions on certain growth parameters during the two growing seasons.

\begin{tabular}{|c|c|c|c|c|c|c|c|c|c|c|c|}
\hline \multicolumn{2}{|c|}{ Treatments } & \multicolumn{2}{|c|}{$\begin{array}{c}\text { Plant height } \\
(\mathrm{cm})\end{array}$} & \multicolumn{2}{|c|}{$\begin{array}{c}\text { Branches } \\
\text { number }\end{array}$} & \multicolumn{2}{|c|}{ leaf number } & \multicolumn{2}{|c|}{$\begin{array}{c}\text { shoot dry } \\
\text { weight }\end{array}$} & \multicolumn{2}{|c|}{$\begin{array}{c}\text { Shoot fresh } \\
\text { weight }\end{array}$} \\
\hline $\begin{array}{l}\text { Water } \\
\text { stress } \\
\% \text { FC } \\
\end{array}$ & $\begin{array}{c}\text { Chitosan } \\
(\mathrm{mg} / \mathrm{l})\end{array}$ & $\begin{array}{c}1 \text { st } \\
\text { season }\end{array}$ & $\begin{array}{c}\text { 2nd } \\
\text { season }\end{array}$ & $\begin{array}{c}1 \text { st } \\
\text { season }\end{array}$ & $\begin{array}{c}2 \mathrm{nd} \\
\text { season }\end{array}$ & $\begin{array}{c}1 \text { st } \\
\text { season }\end{array}$ & $\begin{array}{c}\text { 2nd } \\
\text { season }\end{array}$ & $\begin{array}{l}\text { 1st } \\
\text { eason }\end{array}$ & $\begin{array}{l}\text { 2nd } \\
\text { season }\end{array}$ & $\begin{array}{c}1 \text { st } \\
\text { season }\end{array}$ & $\begin{array}{c}\text { 2nd } \\
\text { season }\end{array}$ \\
\hline \multirow{4}{*}{$\begin{array}{c}70 \% \\
F C\end{array}$} & W & & & $0 \mathrm{c}$ & $\begin{array}{c}4.00 \pm \\
0 \mathrm{c} \\
\end{array}$ & & & \begin{tabular}{|c|}
$7.25 \pm$ \\
$0.052 d$ \\
\end{tabular} & & & \\
\hline & 125 & & & $\begin{array}{c}5.00 \pm \\
0 \mathrm{~b}\end{array}$ & \begin{tabular}{c|}
$4.66 \pm$ \\
$1.154 b$ \\
\end{tabular} & & & \begin{tabular}{|c|}
$7.77 \pm$ \\
$0.121 \mathrm{c}$ \\
\end{tabular} & \begin{tabular}{|c|}
$7.75 \pm$ \\
$0.050 \mathrm{~b}$ \\
\end{tabular} & & \\
\hline & 250 & & & $\begin{array}{c}6.00 \pm \\
0 \mathrm{a} \\
\end{array}$ & & & & \begin{tabular}{|c|}
$8.73 \pm$ \\
$0.692 \mathrm{a}$ \\
\end{tabular} & & & \\
\hline & 500 & & & $\begin{array}{c}4.00 \pm \\
0 c\end{array}$ & $\begin{array}{c}4.00 \pm \\
0 \mathrm{c}\end{array}$ & & & \begin{tabular}{|c|}
$6.88 \pm$ \\
$0.190 \mathrm{e}$ \\
\end{tabular} & \begin{tabular}{|c|}
$6.74 \pm$ \\
$0.144 d$ \\
\end{tabular} & & $\begin{array}{r}36 \\
1.22 \\
\end{array}$ \\
\hline \multirow{4}{*}{$\begin{array}{c}50 \% \\
F C\end{array}$} & W & & & $\begin{array}{c}3.00 \pm \\
0 \mathrm{~d}\end{array}$ & $\begin{array}{c}3.00 \pm \\
0 \mathrm{~d}\end{array}$ & & & & & & \\
\hline & 125 & & & $\begin{array}{c}4.00 \pm \\
0 \mathrm{c}\end{array}$ & $\begin{array}{c}4.00 \pm \\
0 \mathrm{c} \\
\end{array}$ & & & $\begin{array}{l}7.34 \pm \\
0.090 \mathrm{~d} \\
\end{array}$ & \begin{tabular}{|r|}
7.3 \\
0.13 \\
\end{tabular} & & $\begin{array}{r}37 . \\
0.84 \\
\end{array}$ \\
\hline & 250 & & & $\begin{array}{l}5.00 \pm \\
0 \mathrm{~b}\end{array}$ & $\begin{array}{l}5.00 \pm \\
0 \mathrm{~b}\end{array}$ & & & \begin{tabular}{|c|}
$8.22 \pm$ \\
$0.253 \mathrm{~b}$ \\
\end{tabular} & & & \\
\hline & 00 & & & $\begin{array}{l}3.66 \pm \\
1.154 \mathrm{c}\end{array}$ & $\begin{array}{c}3.00 \pm \\
0 \mathrm{~d}\end{array}$ & & $26 \pm$ & \begin{tabular}{|l|}
$6.66 \pm$ \\
$0.046 \mathrm{f}$ \\
\end{tabular} & & & $\begin{array}{c}36.55 \pm \\
0.979 \text { def } \\
\end{array}$ \\
\hline \multirow{4}{*}{$\begin{array}{c}30 \% \\
F C\end{array}$} & 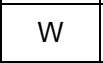 & & $\begin{array}{l}25.33 \pm \\
2.247 i\end{array}$ & $\begin{array}{c}2.00 \pm \\
0 \mathrm{e}\end{array}$ & $\begin{array}{c}2.00 \pm \\
0 \mathrm{e}\end{array}$ & $\begin{array}{c}21 \pm \\
2 j\end{array}$ & $\begin{array}{l}21.33 \pm \\
4.618 \mathrm{~h}\end{array}$ & \begin{tabular}{|c|}
$5.16 \pm$ \\
$0.072 \mathrm{i}$ \\
\end{tabular} & $\begin{array}{l}5.03 \pm \\
0.02 \mathrm{~g}\end{array}$ & $\begin{array}{l}29.41 \pm \\
2.487 \mathrm{i}\end{array}$ & $\begin{array}{l}28.33 \pm \\
5.280 \mathrm{~h}\end{array}$ \\
\hline & 125 & \begin{tabular}{|c|}
$28.90 \pm$ \\
$0.200 \mathrm{gh}$
\end{tabular} & \begin{tabular}{|l|}
$28.10 \pm$ \\
$0.200 \mathrm{~h}$
\end{tabular} & $\begin{array}{c}3.00 \pm \\
0 \mathrm{~d}\end{array}$ & $\begin{array}{c}3.00 \pm \\
0 \mathrm{~d}\end{array}$ & \begin{tabular}{|l|}
$24.33 \pm$ \\
$1.154 \mathrm{~h}$ \\
\end{tabular} & \begin{tabular}{|l|}
$23.33 \pm$ \\
$1.154 \mathrm{~g}$ \\
\end{tabular} & \begin{tabular}{|c|}
$5.97 \pm$ \\
$0.115 \mathrm{~g}$ \\
\end{tabular} & \begin{tabular}{|l|}
$5.77 \pm$ \\
$0.207 \mathrm{f}$
\end{tabular} & & $\begin{array}{l}34.36 \pm \\
2.618 f\end{array}$ \\
\hline & 250 & $\begin{array}{c}39.80 \pm \\
0.346 \mathrm{bc}\end{array}$ & $\begin{array}{l}39.56 \pm \\
1.474 \mathrm{c}\end{array}$ & $\begin{array}{c}4.00 \pm \\
0 \mathrm{c}\end{array}$ & $\begin{array}{c}4.00 \pm \\
0 \mathrm{c} \\
\end{array}$ & $32 \pm$ & \begin{tabular}{|l|}
$31.33 \pm$ \\
$1.154 \mathrm{c}$ \\
\end{tabular} & \begin{tabular}{|c|}
$7.44 \pm$ \\
$0.057 d$ \\
\end{tabular} & $\begin{array}{c}7.42 \pm \\
0.117 c \\
\end{array}$ & $\begin{array}{l}38.52 \pm \\
0.664 d \\
\end{array}$ & $\begin{array}{c}38.17 \pm \\
0.488 \mathrm{~cd}\end{array}$ \\
\hline & 00 & \begin{tabular}{|l|}
$27.53 \pm$ \\
$1.331 \mathrm{hi}$ \\
\end{tabular} & $\begin{array}{l}27.50 \pm \\
0.400 \mathrm{~h}\end{array}$ & $\begin{array}{c}2.66 \pm \\
1.154 \mathrm{~d}\end{array}$ & $\begin{array}{c}2.33 \pm \\
1.154 \mathrm{e}\end{array}$ & \begin{tabular}{|l|}
$22.33 \pm$ \\
$1.154 \mathrm{i}$ \\
\end{tabular} & \begin{tabular}{|l|}
$21.66 \pm$ \\
$1.154 \mathrm{~h}$ \\
\end{tabular} & \begin{tabular}{|c|}
$5.40 \pm$ \\
$0.128 \mathrm{~h}$ \\
\end{tabular} & \begin{tabular}{|c|}
$5.29 \pm$ \\
$0.133 \mathrm{~g}$ \\
\end{tabular} & \begin{tabular}{|l|}
$32.44 \pm$ \\
$3.190 h$ \\
\end{tabular} & $\begin{array}{l}31.53 \pm \\
2.708 \mathrm{~g}\end{array}$ \\
\hline
\end{tabular}

Values are given as mean \pm SD of three replicate. Means in columns by different letters are significantly different at $P<0.05$ by (Duncan's Multiple Range Test). 
The great reduction was observed under severe water stress $(30 \%$ FC). From the same table, it is well noted that foliar application of $\mathrm{CHI}$, in particular, $250 \mathrm{mg} / \mathrm{l}$ improved all plant growth through parameter comparing with untreated control plant. Regarding the interaction effects, the data presented in the same table revealed that application of $\mathrm{CHI}$ at $250 \mathrm{mg} / \mathrm{l}$ significantly increased all growth parameter of cowpea under stress and nonstressed conditions. The only exception was detected at $500 \mathrm{mg} / \mathrm{l}$ which significantly decreased all these parameters. Moreover, application of 125 $\mathrm{mg} / \mathrm{l} \mathrm{CHI} \mathrm{counteracted} \mathrm{the} \mathrm{harmful} \mathrm{effect} \mathrm{of} \mathrm{water} \mathrm{stress} \mathrm{on} \mathrm{plant} \mathrm{growth} \mathrm{due}$ to increasing plant growth under such water stress level.

Chlorophylls and total carbohydrates concentrations:

Data presented in Table (2) indicate that the concentration of total chlorophylls and total carbohydrates were significantly decreased under water stress in both growing seasons as compared with the control plants.

Foliar application of $\mathrm{CHI}$, in particular, $250 \mathrm{mg} / \mathrm{l}$ significantly increased the concentration of both chlorophylls and total carbohydrates as compared with untreated plants under such stress levels. As for its interactions with water stress, the data presented in the same table indicate that chitosan $(500 \mathrm{mg} / \mathrm{l})$ alleviated the harmful effect of moderate and severe water stress in this respect.

Table (2): Effect of water stress and chitosan and their interactions on chlorophyll (mg/g FW) and total carbohydrates (mg/g DW) concentrations during the two growing seasons.

\begin{tabular}{|c|c|c|c|c|c|c|c|c|c|}
\hline \multicolumn{2}{|c|}{ Treatments } & \multicolumn{2}{|c|}{ Chlorophyll A } & \multicolumn{2}{|c|}{ Chlorophyll B } & \multicolumn{2}{|c|}{ Total chlorophylls } & \multicolumn{2}{|c|}{$\begin{array}{c}\text { Total } \\
\text { carbohydrates }\end{array}$} \\
\hline $\begin{array}{l}\text { Water } \\
\text { stress }\end{array}$ & $\begin{array}{c}\text { Chitosan } \\
(\mathrm{mg} / \mathrm{l})\end{array}$ & $\begin{array}{c}\text { 1st } \\
\text { season }\end{array}$ & $\begin{array}{l}\text { 2nd } \\
\text { season }\end{array}$ & $\begin{array}{c}\text { 1st } \\
\text { season }\end{array}$ & $\begin{array}{l}\text { 2nd } \\
\text { season }\end{array}$ & $\begin{array}{c}\text { 1st } \\
\text { season }\end{array}$ & $\begin{array}{c}\text { 2nd } \\
\text { season }\end{array}$ & \begin{tabular}{|c|} 
1st \\
season
\end{tabular} & $\begin{array}{c}\text { 2nd } \\
\text { season }\end{array}$ \\
\hline \multirow{4}{*}{$\begin{array}{l}70 \% \\
\text { FC }\end{array}$} & W & $\begin{array}{c}0.428 \pm \\
0.033 \mathrm{bc}\end{array}$ & $\begin{array}{c}0.507 \pm \\
0.217 \mathrm{bc}\end{array}$ & $\begin{array}{c}0.299 \pm \\
0.008 \mathrm{a}\end{array}$ & $\begin{array}{l}0.195 \pm \\
0.056 \mathrm{a}\end{array}$ & $\begin{array}{c}0.728 \pm \\
0.026 \mathrm{de}\end{array}$ & $\begin{array}{c}0.702 \pm \\
0.165 \mathrm{de}\end{array}$ & $\begin{array}{l}30.18 \pm \\
0.588 \mathrm{e}\end{array}$ & $\begin{array}{l}31.98 \pm \\
1.57 \mathrm{cV}\end{array}$ \\
\hline & 125 & $\begin{array}{l}0.539 \pm \\
0.194 \mathrm{~b}\end{array}$ & $\begin{array}{c}0.516 \pm \\
0.217 \mathrm{bc} \\
\end{array}$ & $\begin{array}{l}0.291 \pm \\
0.218 \mathrm{a} \\
\end{array}$ & $\begin{array}{l}0.293 \pm \\
0.203 \mathrm{a}\end{array}$ & \begin{tabular}{|c|}
$0.814 \pm$ \\
$0.024 \mathrm{bc}$ \\
\end{tabular} & \begin{tabular}{|c|}
$0.809 \pm$ \\
$0.018 \mathrm{bc}$ \\
\end{tabular} & \begin{tabular}{|c|}
$35 \pm$ \\
$1.756 \mathrm{bc}$ \\
\end{tabular} & $\begin{array}{c}36.15 \pm \\
0.515 \mathrm{ab}\end{array}$ \\
\hline & 250 & $\begin{array}{l}0.800 \pm \\
0.225 \mathrm{a}\end{array}$ & $\begin{array}{l}0.724 \pm \\
0.194 \mathrm{a}\end{array}$ & $\begin{array}{l}0.236 \pm \\
0.077 \mathrm{a}\end{array}$ & $\begin{array}{l}0.278 \pm \\
0.090 \mathrm{a}\end{array}$ & $\begin{array}{l}1.037 \pm \\
0.298 \mathrm{a}\end{array}$ & $\begin{array}{l}02 \pm \\
02 \mathrm{a}\end{array}$ & $\begin{array}{l}37.29 \pm \\
0.889 \mathrm{a} \\
\end{array}$ & $\begin{array}{c}38.61 \pm \\
1.66 \mathrm{a}\end{array}$ \\
\hline & 500 & \begin{tabular}{|c|}
$0.462 \pm$ \\
$0.248 \mathrm{bc}$ \\
\end{tabular} & \begin{tabular}{|c|}
$0.394 \pm$ \\
$0.068 \mathrm{bcd}$ \\
\end{tabular} & $\begin{array}{l}0.239 \pm \\
0.261 \mathrm{a} \\
\end{array}$ & $\begin{array}{l}0.284 \pm \\
0.049 \mathrm{a}\end{array}$ & \begin{tabular}{|c|}
$0.702 \pm$ \\
$0.013 \mathrm{def}$
\end{tabular} & $\begin{array}{c}0.679 \pm \\
0.020 \mathrm{de}\end{array}$ & $\begin{array}{l}27.25 \pm \\
4.622 \mathrm{f}\end{array}$ & $\begin{array}{l}30.70 \pm \\
1.39 \mathrm{~cd}\end{array}$ \\
\hline \multirow{4}{*}{$\begin{array}{c}50 \% \\
\text { FC }\end{array}$} & W & \begin{tabular}{|c|}
$0.382 \pm$ \\
$0.180 \mathrm{bc}$ \\
\end{tabular} & \begin{tabular}{|c|}
$0.450 \pm$ \\
$0.263 \mathrm{bc}$ \\
\end{tabular} & $\begin{array}{l}0.253 \pm \\
0.146 \mathrm{a} \\
\end{array}$ & $\begin{array}{l}0.237 \pm \\
0.206 \mathrm{a}\end{array}$ & $\begin{array}{c}0.637 \pm \\
0.035 \mathrm{fg}\end{array}$ & \begin{tabular}{|c|}
$0.596 \pm$ \\
$0.011 \mathrm{fg}$ \\
\end{tabular} & $\begin{array}{l}25.46 \pm \\
0.355 \mathrm{f}\end{array}$ & $\begin{array}{l}30.77 \pm \\
1.25 \mathrm{~cd}\end{array}$ \\
\hline & 125 & \begin{tabular}{|c|}
$0.482 \pm$ \\
$0.351 \mathrm{bc}$
\end{tabular} & $\begin{array}{c}0.576 \pm \\
0.338 \mathrm{ab} \\
\end{array}$ & $\begin{array}{l}0.246 \pm \\
0.344 \mathrm{a}\end{array}$ & $\begin{array}{l}0.150 \pm \\
0.318 \mathrm{a}\end{array}$ & \begin{tabular}{|c|}
$0.729 \pm$ \\
$0.011 \mathrm{de}$
\end{tabular} & $\begin{array}{l}0.727 \pm \\
0.020 \mathrm{~d}\end{array}$ & $\begin{array}{c}31.85 \pm \\
2.276 \mathrm{de}\end{array}$ & $\begin{array}{c}33.55 \pm \\
0.954 \text { bc }\end{array}$ \\
\hline & 250 & \begin{tabular}{|c|}
$0.613 \pm$ \\
$0.237 \mathrm{ab}$
\end{tabular} & $\begin{array}{l}0.725 \pm \\
0.071 \mathrm{a}\end{array}$ & & $\begin{array}{l}46 \pm \\
6 \mathrm{a}\end{array}$ & $\begin{array}{l}78 \pm \\
5 \mathrm{~b}\end{array}$ & $\begin{array}{l}0.872 \pm \\
0.075 \mathrm{~b}\end{array}$ & \begin{tabular}{|c|}
$36.26 \pm$ \\
$0.854 \mathrm{ab}$
\end{tabular} & $\begin{array}{l}37.52 \pm \\
1.060 \mathrm{a}\end{array}$ \\
\hline & 500 & $\begin{array}{l}0.530 \pm \\
0.274 \mathrm{~b}\end{array}$ & $\begin{array}{c}0.401 \pm \\
0.325 \mathrm{bcd}\end{array}$ & $\begin{array}{l}3 \pm \\
4 a\end{array}$ & $\begin{array}{l}0.228 \pm \\
0.310 \mathrm{a}\end{array}$ & $\begin{array}{c}0.674 \pm \\
0.027 \text { efg }\end{array}$ & \begin{tabular}{|l}
$29 \pm$ \\
7 ef
\end{tabular} & $\begin{array}{l}27.28 \pm \\
0.900 f\end{array}$ & $\begin{array}{l}28.87 \pm \\
1.34 \mathrm{de}\end{array}$ \\
\hline \multirow{4}{*}{$\begin{array}{c}30 \% \\
F C\end{array}$} & W & $\begin{array}{l}0.276 \pm \\
0.188 \mathrm{c}\end{array}$ & $\begin{array}{l}0.202 \pm \\
0.058 \mathrm{~d}\end{array}$ & $\begin{array}{l}0.117 \pm \\
0.138 \mathrm{a}\end{array}$ & $\begin{array}{l}0.136 \pm \\
0.033 \mathrm{a}\end{array}$ & $\begin{array}{l}0.394 \pm \\
0.057 \mathrm{i}\end{array}$ & $\begin{array}{l}0.338 \pm \\
0.073 \mathrm{~h}\end{array}$ & $\begin{array}{l}18.86 \pm \\
4.12 \mathrm{~h}\end{array}$ & $\begin{array}{l}19.72 \pm \\
2.72 \mathrm{~g}\end{array}$ \\
\hline & 125 & \begin{tabular}{|c|}
$0.398 \pm$ \\
$0.304 \mathrm{bc}$ \\
\end{tabular} & \begin{tabular}{|c|}
$0.379 \pm$ \\
$0.220 \mathrm{bcd}$ \\
\end{tabular} & $\begin{array}{l}0.200 \pm \\
0.314 \mathrm{a} \\
\end{array}$ & $\begin{array}{l}0.201 \pm \\
0.213 \mathrm{a}\end{array}$ & \begin{tabular}{|c|}
$0.599 \pm$ \\
$0.011 \mathrm{gh}$ \\
\end{tabular} & $\begin{array}{c}0.581 \pm \\
0.019 \mathrm{fg} \\
\end{array}$ & $\begin{array}{l}23.5 \pm \\
2.16 \mathrm{~g}\end{array}$ & $\begin{array}{c}25.77 \pm \\
0.712 \text { ef }\end{array}$ \\
\hline & 250 & $\begin{array}{l}0.546 \pm \\
0.275 \mathrm{~b}\end{array}$ & $\begin{array}{c}0.513 \pm \\
0.234 \mathrm{bc}\end{array}$ & $\begin{array}{l}0.223 \pm \\
0.241 \mathrm{a} \\
\end{array}$ & $\begin{array}{l}0.242 \pm \\
0.264 \mathrm{a}\end{array}$ & $\begin{array}{c}0.769 \pm \\
0.036 \mathrm{~cd} \\
\end{array}$ & \begin{tabular}{|c|}
$0.755 \pm$ \\
$0.042 \mathrm{~cd}$ \\
\end{tabular} & \begin{tabular}{|c|}
$33.33 \pm$ \\
$0.487 \mathrm{~cd}$ \\
\end{tabular} & $\begin{array}{c}35.51 \pm \\
0.401 \mathrm{ab} \\
\end{array}$ \\
\hline & 5 & $\begin{array}{c}0.429 \pm \\
0.274 \mathrm{bc}\end{array}$ & $\begin{array}{c}0.322 \pm \\
0.254 \mathrm{~cd}\end{array}$ & \begin{tabular}{|l|}
$0.113 \pm$ \\
$0.276 \mathrm{a}$
\end{tabular} & $\begin{array}{l}0.201 \pm \\
0.310 \mathrm{a}\end{array}$ & $\begin{array}{c}0.543 \pm \\
0.047 \mathrm{~h}\end{array}$ & $\begin{array}{l}0.524 \pm \\
0.061 \mathrm{~g}\end{array}$ & $\begin{array}{c}21.84 \pm \\
1.79 \mathrm{~g}\end{array}$ & $\begin{array}{c}24.31 \pm \\
1.65 \mathrm{f}\end{array}$ \\
\hline
\end{tabular}

Values are given as mean \pm SD of three replicate. Means in columns by different letters are significantly different at $P<0.05$ by (Duncan's Multiple Range Test). 


\section{lons percentage:}

Water stress is generally recognized as injurious to plants by disturbing the electrolyte balance, resulting in the deficiency of some nutrients. Data presented in Table (3) prove that water stress decreased significantly shoot nitrogen, phosphorus and potassium percentage in both growing seasons. The great reduction occurred under severe water stress. On the other hand, data presented in the same table show that pronounce and highly significant icrease in nitrogen, phosphorous and potassium percentages in the shoot due to exogenous application of $\mathrm{CHI}$, in particular, $250 \mathrm{mg} / \mathrm{l}$ in both growing seasons.

Table (3): Effect of water stress and chitosan and their interactions on nitrogen,phosphorous and potassium percentage during the two growing seasons.

\begin{tabular}{|c|c|c|c|c|c|c|c|}
\hline \multicolumn{2}{|c|}{ Treatments } & \multicolumn{2}{|c|}{ Nitrogen \% } & \multicolumn{2}{|c|}{ Phosphorous \% } & \multicolumn{2}{|c|}{ Potassium\% } \\
\hline $\begin{array}{l}\text { Water } \\
\text { stress } \\
\% \text { FC }\end{array}$ & $\begin{array}{c}\text { Chitosan } \\
\text { (mg/l) }\end{array}$ & 1st season & 2nd season & 1st season & $\begin{array}{l}\text { 2nd } \\
\text { season }\end{array}$ & 1st season & 2nd season \\
\hline \multirow{4}{*}{$70 \% \mathrm{FC}$} & W & $\begin{array}{c}3.71 \pm \\
0.140 \mathrm{f}\end{array}$ & $\begin{array}{c}3.43 \pm \\
0.140 \mathrm{e}\end{array}$ & $\begin{array}{c}0.498 \pm \\
0.008 \mathrm{~cd}\end{array}$ & $\begin{array}{c}0.512 \pm \\
0.011 \mathrm{e}\end{array}$ & $\begin{array}{c}1.25 \pm \\
0.072 \mathrm{e}\end{array}$ & $\begin{array}{c}1.04 \pm \\
0.080 \mathrm{e}\end{array}$ \\
\hline & 125 & $\begin{array}{c}4.29 \pm \\
0.080 \mathrm{c}\end{array}$ & $\begin{array}{c}4.17 \pm \\
0.080 \mathrm{~b}\end{array}$ & $\begin{array}{c}0.539 \pm \\
0.032 \text { bc }\end{array}$ & $\begin{array}{l}0.615 \pm \\
0.045 c\end{array}$ & $\begin{array}{c}1.56 \pm \\
0.034 \mathrm{~b}\end{array}$ & $\begin{array}{c}1.44 \pm \\
0.100 \mathrm{~b}\end{array}$ \\
\hline & 250 & $\begin{array}{c}4.76 \pm \\
0.140 \mathrm{a}\end{array}$ & $\begin{array}{c}4.48 \pm \\
0.140 \mathrm{a}\end{array}$ & $\begin{array}{l}0.725 \pm \\
0.161 \mathrm{a}\end{array}$ & $\begin{array}{l}0.762 \pm \\
0.032 \mathrm{a}\end{array}$ & $\begin{array}{c}1.68 \pm \\
0.023 \mathrm{a}\end{array}$ & $\begin{array}{c}1.59 \pm \\
0.050 \mathrm{a}\end{array}$ \\
\hline & 500 & $\begin{array}{c}3.38 \pm \\
0.161 \mathrm{~g}\end{array}$ & $\begin{array}{l}3.22 \pm \\
0.140 \mathrm{f}\end{array}$ & $\begin{array}{c}0.478 \pm \\
0.017 \mathrm{de}\end{array}$ & $\begin{array}{c}0.493 \pm \\
0.018 \text { ef }\end{array}$ & $\begin{array}{c}1.10 \pm \\
0.113 f\end{array}$ & $\begin{array}{l}0.913 \pm \\
0.050 f\end{array}$ \\
\hline \multirow{4}{*}{$50 \%$ FC } & W & $\begin{array}{c}3.12 \pm \\
0.080 \mathrm{~h}\end{array}$ & $\begin{array}{c}3.01 \pm \\
0.140 \mathrm{gh}\end{array}$ & $\begin{array}{c}0.428 \pm \\
0.016 \text { efg }\end{array}$ & $\begin{array}{c}0.449 \pm \\
0.017 \mathrm{~g}\end{array}$ & $\begin{array}{c}0.85 \pm \\
0.070 \mathrm{~h}\end{array}$ & $\begin{array}{c}0.78 \pm \\
0.034 \mathrm{~h}\end{array}$ \\
\hline & 125 & $\begin{array}{c}3.99 \pm \\
0.140 \mathrm{e}\end{array}$ & $\begin{array}{c}3.64 \pm \\
0.242 \mathrm{~d}\end{array}$ & $\begin{array}{c}0.514 \pm \\
0.011 \mathrm{~cd}\end{array}$ & $\begin{array}{l}0.518 \pm \\
0.024 \mathrm{e}\end{array}$ & $\begin{array}{c}1.37 \pm \\
0.100 \mathrm{~d}\end{array}$ & $\begin{array}{c}1.19 \pm \\
0.092 \mathrm{~d}\end{array}$ \\
\hline & 250 & $\begin{array}{c}4.50 \pm \\
0.213 b\end{array}$ & $\begin{array}{c}4.29 \pm \\
0.080 \mathrm{~b}\end{array}$ & $\begin{array}{l}0.592 \pm \\
0.043 \mathrm{~b}\end{array}$ & $\begin{array}{l}0.705 \pm \\
0.051 b\end{array}$ & $\begin{array}{c}1.64 \pm \\
0.050 \mathrm{a}\end{array}$ & $\begin{array}{c}1.54 \pm \\
0.050 \mathrm{a}\end{array}$ \\
\hline & 500 & $\begin{array}{c}3.19 \pm \\
0.080 \mathrm{~h}\end{array}$ & $\begin{array}{c}3.10 \pm \\
0.080 \mathrm{fg}\end{array}$ & $\begin{array}{c}0.460 \pm \\
0.030 \text { def }\end{array}$ & $\begin{array}{c}0.470 \pm \\
0.011 \mathrm{fg}\end{array}$ & $\begin{array}{c}0.97 \pm \\
0.072 \mathrm{~g}\end{array}$ & $\begin{array}{l}0.836 \pm \\
0.050 \mathrm{~g}\end{array}$ \\
\hline \multirow{4}{*}{$30 \% \mathrm{FC}$} & W & $\begin{array}{c}2.68 \pm \\
0.080 \mathrm{k}\end{array}$ & $\begin{array}{l}2.49 \pm \\
0.213 i\end{array}$ & $\begin{array}{c}0.369 \pm \\
0.049 \mathrm{~g}\end{array}$ & $\begin{array}{l}0.378 \pm \\
0.029 \mathrm{i}\end{array}$ & $\begin{array}{l}0.703 \pm \\
0.080 j\end{array}$ & $\begin{array}{l}0.626 \pm \\
0.057 \mathrm{j}\end{array}$ \\
\hline & 125 & $\begin{array}{l}3.01 \pm \\
0.14 \mathrm{i}\end{array}$ & $\begin{array}{c}2.87 \pm \\
0.140 \mathrm{~h}\end{array}$ & $\begin{array}{c}0.404 \pm \\
0.016 \mathrm{fg}\end{array}$ & $\begin{array}{l}0.420 \pm \\
0.020 \mathrm{~h}\end{array}$ & $\begin{array}{c}0.813 \pm \\
0.050 \mathrm{hi}\end{array}$ & $\begin{array}{c}0.73 \pm \\
0.034 \mathrm{hi}\end{array}$ \\
\hline & 250 & $\begin{array}{c}4.17 \pm \\
0.080 \mathrm{~d}\end{array}$ & $\begin{array}{c}3.92 \pm \\
0.280 \mathrm{c}\end{array}$ & $\begin{array}{c}0.499 \pm \\
0.131 \mathrm{~cd}\end{array}$ & $\begin{array}{l}0.547 \pm \\
0.045 \mathrm{~d}\end{array}$ & $\begin{array}{c}1.49 \pm \\
0.100 \mathrm{c}\end{array}$ & $\begin{array}{c}1.30 \pm \\
0.072 \mathrm{c}\end{array}$ \\
\hline & 500 & $\begin{array}{c}2.84 \pm \\
0.213 \mathrm{j}\end{array}$ & $\begin{array}{c}2.63 \pm \\
0.224 \mathrm{i}\end{array}$ & $\begin{array}{l}0.393 \pm \\
0.008 \mathrm{~g}\end{array}$ & $\begin{array}{c}0.400 \pm \\
0.010 \mathrm{hi}\end{array}$ & $\begin{array}{l}0.753 \pm \\
0.023 \mathrm{ij}\end{array}$ & $\begin{array}{l}0.696 \pm \\
0.023 \mathrm{i}\end{array}$ \\
\hline
\end{tabular}

Values are given as mean \pm SD of three replicate. Means in columns by different letters are significantly different at $\mathbf{P}<0.05$ by (Duncan's Multiple Range Test).

It has been observed that any of $\mathrm{CHI}$, in particular, $250 \mathrm{mg} / \mathrm{l}$ alleviated the harmful effect of water stress especially at high level on nitrogen, phosphorous and potassium percentages.

\section{Leaf anatomy}

Cross section of $3^{\text {rd }}$ terminal leaflet of cowpea showed that there were significant changes in leaf anatomical characteristics due to water stress (Table 4 and figure, 1). In particular, water stress resulted in a significant decrease of the thickness of almost all anatomical characters i.e. 
components of the mesophyll, as well as of the entire lamina thickness (Table 4 and figure, 1). Data presented in Table (4) and illustrated in Figure (1) indicate that water stress decreased thickness of leaflet and mesophyll tissue as well as main vascular bundle dimensions, xylem and phloem tissue thickness.

The data also indicated that cowpea leaflet has a well developed layer of water storage tissue which consists of 1-3 cell thick laying under main vascular bundle in the midrib region. Foliar application of chitosan,in particular, $250 \mathrm{mg} / \mathrm{l}$ increased the thickness of cowpea leaf blade, due to the increase in the thickness of mesophyll tissue. In addition, the thickness of leaf blade through midrib region was also increased, due to the increase in the midrib vascular bundle. Moreover, chitosan application increased the thickness of water storage tissue thickness. chitosan resulted in increasing the area of xylem and phloem tissues, due to the stimulation of pro-cambium activity in the midrib bundle during their differentiation. Concerning the interaction between water stress and chitosan, the interactions increased the cowpea leaflet anatomical characteristics as compared with untreated plants under such stress levels.

Table (4): Effect of water stress and chitosan and their interactions on leaflet anatomical characters of cowpea plants in the second season.

\begin{tabular}{|c|c|c|c|c|c|c|c|c|c|c|}
\hline \multicolumn{2}{|c|}{ Treatments } & \multirow{2}{*}{ 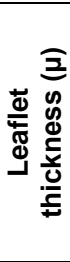 } & \multirow{2}{*}{ 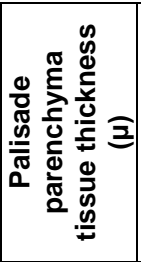 } & \multirow{2}{*}{ 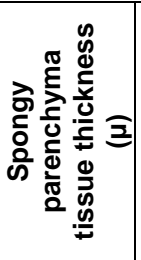 } & \multirow{2}{*}{ 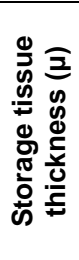 } & \multirow{2}{*}{ 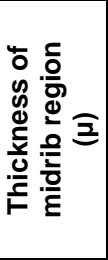 } & \multicolumn{2}{|c|}{\begin{tabular}{|c|} 
Main \\
vascular \\
bundle \\
dimensions \\
$(\mu)$ \\
\end{tabular}} & \multirow{2}{*}{ 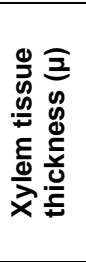 } & \multirow{2}{*}{ 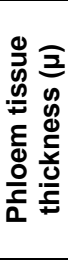 } \\
\hline $\begin{array}{c}\text { Water } \\
\text { stress } \\
\% \text { FC }\end{array}$ & $\begin{array}{c}\text { Chitosan } \\
(\mathrm{mg} / \mathrm{l})\end{array}$ & & & & & & length & width & & \\
\hline \multirow{4}{*}{$\begin{array}{c}70 \% \\
\text { FC }\end{array}$} & W & 68 & 32 & 36 & 16 & 276 & 48 & 96 & 36 & 12 \\
\hline & 125 & 68 & 36 & 32 & 24 & 328 & 60 & 104 & 44 & 16 \\
\hline & 250 & 68 & 36 & 32 & 44 & 336 & 60 & 116 & 40 & 20 \\
\hline & 500 & 56 & 32 & 24 & 32 & 284 & 52 & 112 & 32 & 20 \\
\hline \multirow{4}{*}{$50 \% \mathrm{FC}$} & $\mathrm{W}$ & 52 & 28 & 24 & 24 & 216 & 32 & 68 & 24 & 8 \\
\hline & 125 & 72 & 40 & 32 & 44 & 308 & 52 & 124 & 36 & 16 \\
\hline & 250 & 72 & 36 & 36 & 52 & 316 & 56 & 128 & 40 & 16 \\
\hline & 500 & 56 & 32 & 24 & 40 & 316 & 56 & 92 & 44 & 12 \\
\hline \multirow{4}{*}{$30 \% \mathrm{FC}$} & W & 48 & 24 & 24 & 16 & 188 & 28 & 60 & 20 & 8 \\
\hline & 125 & 56 & 36 & 20 & 28 & 268 & 36 & 88 & 20 & 16 \\
\hline & 250 & 60 & 28 & 32 & 44 & 324 & 48 & 96 & 32 & 16 \\
\hline & 500 & 52 & 28 & 24 & 20 & 212 & 36 & 68 & 24 & 12 \\
\hline
\end{tabular}

Since leaves are the main organs of internal water removal, $\mathrm{CHI}$ treated plants under normal or stressed conditions undertaken leaf anatomical alterations in order to save water. In fact, cowpea leaves treated with $\mathrm{CHI}$ showed a thicker entire leaf lamina and palisade mesophyll than untreated plants under such stress levels. A thicker palisade parenchyma in this treatments may enhance survival and growth under water stress (WS) conditions by improving water relations and providing higher protection for the inner tissues (Bacelar et al. 2006). 

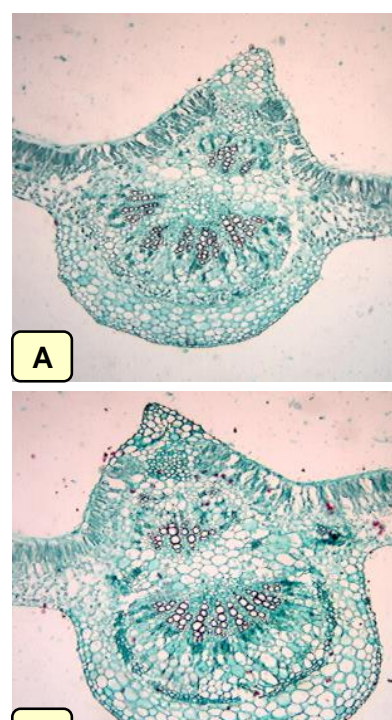

D
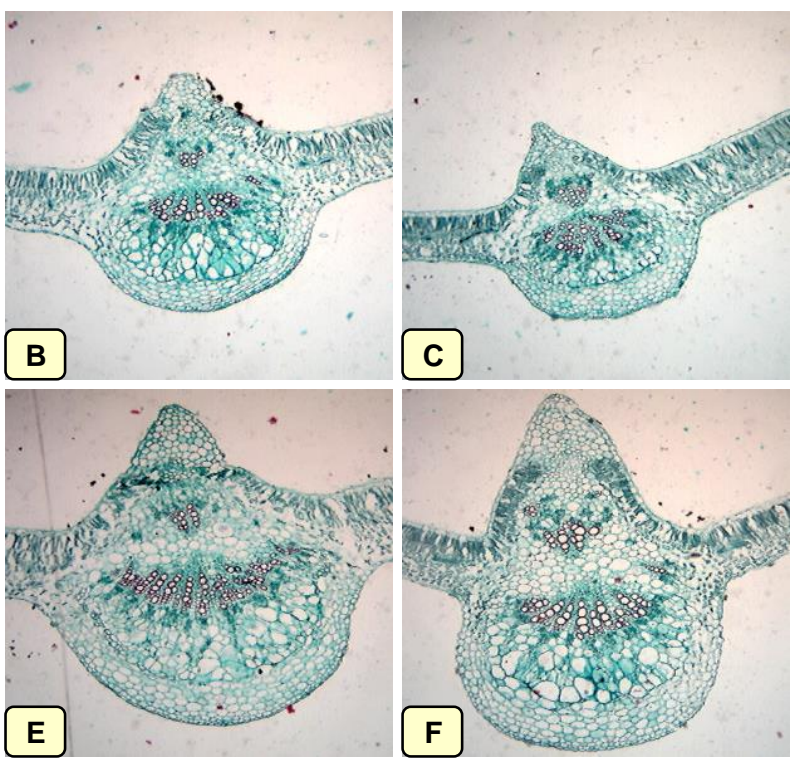

Figure (1): Effect of water stress and chitosan and their interactions on leaflet anatomical characters of cowpea plants in the second season (A, $70 \%$ of field capacity; B, $50 \%$ of field capacity; C, $30 \%$ of field capacity; D, $70 \%$ FC $+250 \mathrm{mg} / \mathrm{l}$ chitosan; E, $50 \%$ FC +250 mg/l chitosan; E, 30\% FC +250 mg/l chitosan).

Yield and its components:

Although water deficit affects all stages of the growth and development of crops, pod yield is much more depressed by water deficit than vegetative growth. In this concern, data presented in Table (5) show that pod yield per plant (g/plant) and seed quality (Nitrogen, phosphorous, potassium, protein and carbohydrates percentages) were significantly decreased with an increase in water deficit. On the other hand, foliar application of $\mathrm{CHI}$ concentrations, in particular, $250 \mathrm{mg} / \mathrm{l}$ resulted in a highest increase in cowpea yield and improved seed quality. The interaction treatments indicated that application of $250 \mathrm{mg} / \mathrm{l} \mathrm{CHI}$ under moderate and severe water deficit significantly increased the yield and its quality. Meanwhile, the lowest and highest concentrations from $\mathrm{CHI}$ under moderate and severe water stress alleviated the harmful effect of water deficit in this respect. 
Farouk, S. and Amany R. Abd EL Mohsen 


\section{DISCUSSION}

The inhibiting effects of water stress on plant growth were previously supported by Abdalla (2011), Vurayai et al (2011) and Hefny (2011) using soybean, bambara groundnuts and white lupin plants respectively. It is well known that, water stress conditions, causes a multitude of changes in molecular, biochemical and physiological phenomena, thereby affecting plant growth and development (Boutraa 2010). Such decline in plant growth in response to water stress might be due to either decrease in cell elongation resulting from the inhibiting effect of water shortage on growth promoting hormones which, in turn, led to a decrease in each of cell turgor, cell volume and eventually cell growth (Banon et al. 2006), and/ or due to blocking up of xylem and phloem vessels thus hindering any translocation through. Moreover, water stress conditions cause a marked suppression in plant photosynthetic efficiency, mainly due to closing of stomata, which limits $\mathrm{CO}_{2}$ diffusion into the leaf, or due to inhibition in Ribulose-1,5-bisphosphate carboxylase/oxygenase (Ribusco), a non-stomatal factor (Lawlor and Cornic 2002) and impairment of ATP synthesis (Tezara et al. 1999). Also, the depression effect of water stress on growth parameters may be attributed to drop in leaf relative water content in which reduce the leaf turgor (unpublished data), the assimilation of water and nitrogen compounds (Reddy et al. 2003), which affects the rate of cell division and enlargement. In this concern, a reduction in vegetative growth of plants under drought, in particular shoot growth, reduced cyclin-dependent kinase activity resulting in slower cell division as well as inhibition of growth and/or due to the relatively severe reduction pertaining to plant tissue, cell size, number of cell per unit or intercellular space (EL-Beltagy et al. 1984). Also, drought stress reduced the uptake of essential elements and photosynthetic capacity reduction (Kandil et al. 2001) as well as the excessive accumulation of intermediate compounds such as reactive oxygen species (Yazdanpanah et al. 2011) which cause oxidative damage to DNA, lipid and proteins and consequently a decrease in plant growth. Finally, water stress leads to increase in abscisic acid levels in roots, which will transport from roots to shoot and will act in the apical region of the plant with antagonist of the auxins and cytokinins, responsible for growth and cell division, respectively (Abdalla 2011) as well as inhibit DNA synthesis. On contrast, foliar spraying of chitosan in both seasons showed in most cases, a significant increase in cowpea growth parameters under normal or stressed conditions, the effect was more pronounced with chitosan at $250 \mathrm{mg} / \mathrm{l}$. This result was supported by Chibu and Shibayama (1999) who revealed that dry weights of dry land rice c.v. Misatohatamochi grown with both 0.1 and $0.5 \%$ of $\mathrm{CHI}$ were increased. Similar results were reported by Ghoname et al. (2010) on sweet pepper and Farouk et al. $(2008,2011)$ on cucumber and radish plants. The stimulating effect of $\mathrm{CHI}$ on plant growth may be attributed to an increase in the availability and uptake of water and essential nutrients through adjusting osmotic pressure in plant cells and reducing the accumulation of harmful free radicals through its effect on increasing the antioxidants compound and enzymes activities (Guan et al. 
2009). Khan et al. (2002) added that foliar application of $\mathrm{CHI}$ increased net photosynthetic rate $\left(\mathrm{P}_{\mathrm{N}}\right)$ of soybean and maize, this increase was correlated with increase in stomatal conductance $\left(g_{s}\right)$ and transpiration rate $(E)$, without any effects on intercellular $\mathrm{CO}_{2}$ concentration $\left(\mathrm{C}_{\mathrm{i}}\right)$. The increase in $\mathrm{P}_{\mathrm{N}}$ and $\mathrm{g}_{\mathrm{s}}$ in the absence of any increase in $\mathrm{C}_{i}$ indicates that the increase in $\mathrm{P}_{\mathrm{N}}$ is due to enhanced uptake of $\mathrm{CO}_{2}$ within the leaf that results in improved $\mathrm{g}_{\mathrm{s}}$, rather than due to more open stomata leading to increased $\mathrm{P}_{\mathrm{N}}$. If an increase in stomatal aperture had been the primary cause of the increase in $P_{N}$, an increase in the leaf $C_{i}$ would have been expected (Morison 1998). In addition, the positive effect of $\mathrm{CHI}$ on plant growth may be due to its effect on increasing phosphorous content as presented in the present investigation. Phosphorous is an essential nutrient and plays an important role in the biosynthesis and translocation of carbohydrates and is necessary in stimulating cell division and the formation of DNA and RNA (Nijjar 1985)

Regarding the concentrations of chlorophylls and carbohydrates, the present investigation proved that water stress decreased chlorophylls and total carbohydrates concentration. The decrease in chlorophyll content under drought is thus a commonly observed phenenmene (Nikolaeva et al. 2010, Kumar et al. 2011). The decrease in chlorophyll under water stress might be due to reduced synthesis of the main chlorophyll pigment complexes encoded by the cab gene family (Allakhverdiev et al. 2003) or destruction of chiral macro-aggregates of light harvesting chlorophyll ' $a$ ' or ' $b$ ' pigment protein complexes (CHClls) which protect the photosynthetic apparatus or due to oxidative damage of chloroplast lipids, pigments and proteins (Lai et al. 2007). In addition, it may be due to impairment in the supply of magnesium and iron to the leaves. Similarly, the reduction in total sugar content induced by water stress treatments may be due to its inhibitory effect on photosynthetic activities, photosynthetic pigment concentrations (Table, 2) as well as on the activity of ribulose diphosphate carboxylase leading to decrease in all sugar fractions (Stibrova et al. 1986). As regard to, the effects of chitosan especially at $250 \mathrm{mg} / \mathrm{l}$ on increasing chlorophylls and total carbohydrates contents were confirmed with Farouk et al. (2008 on cucumber plant) and Farouk et al. (2011 on radish plant). The influence of $\mathrm{CHI}$ on alleviating the water stress effect on the photosynthetic pigments might be due to the fact that $\mathrm{CHI}$ enhanced the endogenous level of cytokinins, which stimulates chlorophyll synthesis. Chibu and Shiayama (2001) referred these positive effects to more availability of amino compounds released from $\mathrm{CHI}$. Data in the present investigation indicate that foliar application of $\mathrm{CHI}$ increased significantly both nitrogen and potassium content in plant shoot (Table, 3) which may be play an important role in increasing the number of chloroplast per cell, the cell size and number per unit area as well as increased the synthesis of chlorophyll (Possingham 1980).

Water stress affects the availability of nutrients in the soil by its effects on the solubility and precipitation of salt and alters physiological processes within the plant, including nutrient uptake and translocation (Power 1990). Nutrient uptake by plants is generally decreased under water stress conditions owing to a substantial decrease in transpiration rates and impaired active transport and membrane permeability (Levitt 1980), and resulting in a 
reduced root-absorbing power of crop plants. Nitrogen serves as constituents of many plant cell components like amino and nucleic acids. Therefore, $\mathrm{N}$ deficiency rapidly inhibits plant growth. The decrease in $\mathrm{N}$ content due to water stress has been reported in various crops including wheat (Singh and Usha 2003) and in soybean (Tanguilig et al. 1987). Phosphorous is one of the most important nutrients in the growth and development of plants. It plays a key role in cellular energy transfer, respiration, photosynthesis. Phosphorous uptake decreased with decreasing soil moisture in different crops like pepper (Turner 1985) and wheat (Ashraf et al. 1998). The role of $\mathrm{CHI}$ on increasing ion contents may be due to its effects on stabilizing cellular membrane through increasing the antioxidants substances and saving cell membrane from oxidative stress so improved plant cell permeability (unpublished data) leading to increasing ion content. This observation was supported with the results of Guan et al. (2009) who indicate that application of CHI significantly decreased lipid peroxidation, due to stimulation of some antioxidants enzymes leading to decreasing membrane permeability and improved its functions. More reports confirmed these results i.e. Farouk et al. (2008 and 2011) and Ghoname et al. (2010).

The reduction in cowpea yield due to water stress was confirmed with Costa et al. (2008), Vurayai et al. (2011) and Hefny (2011). In legume plants like cowpea, seed yield is determined by the production of three components; the number of pods per plant that reach maturity, the average number of seeds in each pod and dry weight of seeds. There are many hypothesis show the influence of water stress on yield. One of them, proved that water stress may be decreased number of branches and leaves per plant (Table, 1) as well as leaf area as indicate in this study (unpublished data), resulting in a reduction in the supply of carbon assimilate and photosynthetic rate as well as reduces radiation interception by plants and consequently less biomass produced as well as decreased translocation of photoassimulate towards the developing fruits (Kumar et al. 1994). Another possibility to reduce the yield due to water stress is increasing the rate of flower and pod abortion (Liu et al. 2003). A decreased rate of carbohydrates flux from leaves to reproductive structures has been reported to controlling pod set in well watered plants (Kokubun et al. 2001, Setter et al. 2001). Recent evidence supports this hypothesis. In maize, low water potential disrupt carbohydrates metabolism in ovaries by reducing the activity of acid invertase, which is the key enzymes catalyzing breakdown of incoming sucrose during ovary and early seed development (Anderson et al. 2002). Moreover, Song et al. (1998) showed that water stress, induced swollen pollen and filament development, decreased filament fertility and resulted in reeducation in grain number and weight per ear. However, the increase in cowpea yield due to $\mathrm{CHI}$ application may be due to its effects on stimulation of physiological processes which reflect on improving vegetative growth that followed by active translocation of the photoassimilates from source to sink in cowpea plant due to increasing leaf blade thickness as well as dimensions of vascular bundles as indicated from the present investigation. The increased in plant biomass may be due to improving photosynthetic machinery (Khan et al. 2002). These results were supported by Ghoname et al. (2010) who observed that foliar application of 
$\mathrm{CHI}$ on sweet pepper increased significantly no. of fruits per plant and mean weight of fruit as well as some quality characteristics like total acidity, total soluble solid and ascorbic acid content in the fruit. The role of $\mathrm{CHI}$ on alleviating the harmful effect of water stress on yield may be due to an increase in stomatal conductance and net photosynthetic $\mathrm{CO}_{2}$ fixation activity under water stress (Khan et al. 2002) and to its role as antitranspiration to save water. $\mathrm{CHI}$ treated plants showed a thicker entire leaf lamina, upper epidermis and palisade mesophyll than untreated plants under such stress levels. A thicker upper epidermis (including upper cuticle) and a thicker palisade parenchyma in this treatments may enhance survival and growth under water stress (WS) conditions by improving water relations and providing higher protection for the inner tissues (Bacelar et al. 2006) which leading to increasing plant yield.

It could be concluded that treated cowpea plants with chitosan inducedits ability to grow under water stress conditions. It may be explaind that cowpea plants with chitosan can produce some metabolites which cause closure of the stomata resulting in reduction of the transpiration.

Acknowledgement; We would to thanks all staff members of Agric. Botany Department, Faculty of Agriculture, Mansoura University, Egypt, in particular, Prof M.N. Helali for his helpful insights and critical review of the manuscript.

\section{REFERENCES}

Abdalla, M.M. (2011). Beneficial effects of diatomite on the growth, the biochemical contents and polymorphic DNA in Lupinus albus plants grown under water stress. Agriculture and Bology Jurnal of Nrth America 2: 207-220.

Abdel-Mawgoud, A.M.R.; A.S. Tantawy; M.A. El-Nemr and Y.N. Sassine (2010). Growth and yield responses of strawberry plants to chitosan application. European Journal of Scientific Research 39 (1):161-168.

Allakhverdiev, I.; H. Hayashi; Y. Nishiyama; A.G. Ivanov; J.A. Aliev; V.V. Klimov; N. Murata and R. Carpentier (2003). Glycinebetaine protects the D1/D2/Cytb559 complex of photosystem II against photo-induced and heat-induced inactivation. J Plant Physiol 160:41-49.

Anderson, M.N.; F. Asch; Y. Wu; C.R. Jnsen, H. Naested; V.O. Mogensen and K.E. Koch (2002). Soluble invertase expressionis an early target of drought stress during the critical, abortion-sensetive phase of young ovary development in maize. Plant Physiology 130: 591-604.

Ashraf, M.Y.; S.A. Ali and A.S. Bhatti (1998). Nutritional imblances in wheat (Triticum aestivum L.) genotypes grown at soil water stress. Acta Physiol Plant. 20 (3):307-310.

Banon, S.J.; J. Ochoa; J.A. Franco; J.J. Alarcon and M.J. Sanchez-Blanco (2006). Hardening of coleander seedlings by deficit irrigation and low air humidity. Environ Exp Bot 56:36-43. 
Bacelar, E.A.; D.L. Santos; J.M. Moutinho-Pereira; B.C. Goncalves; H.F. Ferreira and C.M. Correia (2006). Immediate response and adaptative strategies of three olive cultivars under contrasting water availability regimes: changes on structure and chemical composition of foliage and oxidative damage. Plant Sci. 170:596-605

Bittelli, M.; M. Flury; G.S. Campbell and E.J. Nichols (2001). Reduction of transpiration through foliar application of chitosan. Agricultural and Forest Meteorology 107(3): 167-175

Boutraa, T. (2010). Improvement of water use efficiency in irrigated agriculture: A review. J Agron 9:1-8.

Carvalho, I.S.; C.P. Ricardo and M. Chaves (2004). Quality and Distribution of Assimilates within the Whole Plant of Lupines ( $L$. albus and $L$. mutabilis) Influenced by Water Stress. J Agron Crop Sci 190: 205-210.

Chibu, H. and H. Shibayama (2001). Effects of chitosan applications on the growth of several crops. In: Uragami T, Kurita K, Fukamizo T(eds) Chitin and Chitosan in Life Science, Yamaguchi, pp 235-239.

Chibu, H. and H. Shibayama (1999). Effects of chitosan applications on the early growth of several crops. Report of Kyushu Branch of the Crop Science Society of Japan 65: 83-87.

Cooper, T.G. (1977). The tools of biochemistry. A Wiley-Interscience Pub. John Wiley and Sons, New York.

Costa, R.C.L.;A.K.S. Lobato; C.F. Oliveira Neto; P.S.P. Maia; G.A.R. Alves and H.D. Laughinghouse (2008). Biochemical and physiological responses in two Vigna unguiculata (L.) Walp. cultivars under water stress. J Agron 7: 98-101.

Doares, S.H.; T. Syrovets; E.W. Wieler and A. Ryan (1995). Oligogalacturonides and chitosan activate plant defensive gene through the octadecanoid pathway. PNAS 92:4095-4098.

EL-Beltagy, A.S.; M.T. EL-Said; M.H. Sawsan; M.G. Hasniya and A. Abo ELMaksoud (1984). Effect of different water regimes on tomato. Ann Agric Sci Ain Shams Univ 29:1937-1956.

Farouk, S.; A.A. Mosa; A.A. Taha; Heba M. Ibrahim and A.M. EL-Gahmery (2011). Protective Effect of Humic acid and Chitosan on Radish (Raphanus sativus L. var. sativus) Plants Subjected to Cadmium Stress. Journal of Stress Physiology and Biochemistry 7(2):99-116

Farouk, S.; K.M. Ghoneem and Abeer A. Ali (2008). Induction and Expression of systematic risistance to downy mildew disease in cucumber plant by elicitors. Egyptian Journal of Phytopathology vol (1-2): 95-111.

Gaballah, M.S. and M. Moursy (2004). Reflectants application for increasing wheat plant tolerance against salt stress. Pak J Biol Sci 7:956-962.

Ghoname, A.A.; M.A. EL-Nemr; A.M.R. Abdel-Mawgoud and W.A. ELTohamy (2010). Enhancement of sweet pepper crop growth and production by application of biological, organic and nutritional solutions. Res J Agric Biol Sci 6(7): 349-355.

Guan Ya-jing, Jin Hu, Xian-ju Wang and Chen-xia Shao (2009) Seed priming with chitosan improves maize germination and seedling growth in relation to physiological changes under low temperature stress. Journal of Zhejiang University Science B 10(6):427-433. 
Hefny, M.M. (2011). Agronomical and Biochemical Responses of White Lupinus albus L. Genotypes to Contrasting Water Regimes and Inoculation Treatments. Journal of American Science 7(3):187-198

Jansen, G. (2008). Effects of temperature on yield and protein content of Lupinus angustifolius cultivars. In: proceedings of Lupins for health and wealth. $12^{\text {th }}$ International lupin conference. 14-18 September 2008. Fremantle, Western Australia.

Kalra, Y.P. (1998). Handbook of Reference Methods for Plant Analysis. CRC Press, Boca Raton, Florida, USA.

Kandil, S.A.; M.S.A. Abo-El-Kheir and H.A. El-Zeiny (2001). Response of some wheat cultivars to water stress imposed at certain growth stages. Egyptian J Appl Sci 16: 82-98

Yazdanpanah, S.; A. Baghizadeh and F. Abbassi (2011). The interaction between drought stress and salicylic and ascorbic acids on some biochemical characteristics of Satureja hortensis. African Journal of Agricultural Research 6(4): 798-807

Khan, M.H.; K.L.B. Singha and S.K. Panda (2002). Changes in antioxidant levels in Oryza sativa L. roots subjected to $\mathrm{NaCl}$ salinity stress. Acta Physiol Plant 24:145-148.

Kokubun, M.; S. Shimada and M. Takahashi (2001) Flower abortion caused by preanthesis water deficit is not attributed to impairment of pollen in soybean. Crop Science 41:1517-1521.

Kumar, A.; D.P. Singh and P. Singh (1994). Influence of water stress on photosynthesis, transpiration, water use efficiency and yield of Brassica juncea L. Field Crops Research 37:95-101.

Kumar Ravi Ranjan, Krishna Karajol and G.R. Naik (2011). Effect of Polyethylene Glycol Induced Water Stress on Physiological and Biochemical Responses in Pigeonpea (Cajanus cajan L. Millsp.). Recent Research in Science and Technology 3(1):148-152.

Lai, Q.; B. Zhi-yi; Z. Zhu-Jun; Q. Qiong-Qiu and M. Bi-Zeng (2007). Effects of Osmotic stress on antioxidant enzymes activities in leaf discs of PSAG12-IPT modified gerbera. 8(7):458-464.

Lawlor, D.W. and G. Cornic (2002). Photosynthetic carbon assimilation and associated metabolism in relation to water deficits in higher plants. Plant Cell Environ 25:275-294

Leung, J. and J. Giraudat (1998). Abscisic acid and signal transduction. Annu Rev Plant Physiol Plant Mol Biol 49:199-222.

Levitt, J. (1980). Responses of plants to environmental stresses. $2^{\text {nd }}$ ed., New York, Acad. Press.

Lichtenthaler, H.K. and A.R. Wellbum (1985). Determination of total carotenoids and chlorophylls A and B of leaf in Different Solvents. Biol Soc Trans 11:591-592.

Liu, F.; M.N. Andersen and C.R. Jensen (2003). Loss of pod set caused by drought stress is associated with water status and ABA content of reproductive structures in soybean. Funct Plant Biol 30:271-280. 
Lobato, A.K.S.; R.C.L. Costa and C.F. Oliveira Neto (2006). NR activity and RWC in Feijao-Caupi under water stress. In Proceedings of the 1st Congress Nacional de Feijao-Caupi and 6th Reuniao Nacional de Feijao-Caupi,22 - 26 May, Teresina, Brasil Empresa Brasileira de Agropecuaria, Teresina.

Lobato, A.K.S.; C.F. Oliveira Neto; R.C.L. Costa; B.G. Santos Filho; F.J.R. Cruz and H.D. Laughinghouse (2008). Biochemical and Physiological behaviour of Vigna unguiculata (L) Walp, under water stress during the vegetative phase. Asian J Plant Sci 7(1): 44-49.

Morison, J.I.L. (1998). Stomatal response to increased CO2 concentration. J Exp Bot 49:443-452.

Mullet, J.E. and M.S. Whitsitt (1996). Plant cellular responses to water deficit. Plant Growth Regulation. 20 (2):119-124.

New, N.; S. Chandrkrachang and W.F. Stevens (2004). Application of chitosan in Myanmar's agriculture sector, in: Proceedings of the Sixth Asia Pacific Chitin and Chitosan Symposium, May23-26, The National Universityof Singapore, Singapore.

Nijjar, G.S. (1985). Nutrition of fruit trees. Usha Raji Kumar, Kalyani, New Delhi, India

Nikolaeva, M.K.; S.N. Maevskaya; A.G. Shugaev and N.G. Bukhov (2010). Effect of drought on chlorophyll content and antioxidant enzyme activities in leaves of three wheat cultivars varying in productivity. Rus J Plant Physiol 57:87-95.

Ogbonnaya, C.I.; B. Sarr; C. Brou; O. Diouf; N.N. Diop and H. Roy-Macauly (2003). Selection of cowpea in hydroponics, pots and field for drought tolerance. Crop Sci 43:1114-1120.

Possingham, J.V. (1980). Plastid replication and development in the life cycle of higher plants. Annu Rev Plant Physiol 31:113-129.

Power, J.F. (1990). Role of moisture stress in plant nutritional function in crops as enhancers of nutrient use. Acad. Press, New York

Reddy, T.Y.; V.R. Reddy and N. Anbumozhi (2003). Physiological responses of grounfnut (Arachis hypogaea L.) to drought stress and its amelioration: critical review. Plant growth Reg 41(3):75-78

Sadasivam, S. and A. Manickam (1996) Biochemical methods, $2^{\text {nd }}$ edition, New age international. India.

Setter, T.L.; B.A. Flannigan and J. Melkonian (2001). Loss of kernel set due to water deficit and shade in maize: carbohydrate supplies, abscisic acid, and cytokinins. Crop Sci 41:1530-1540.

Shao, H.; C. Li-Ye; C. Abdul Jaleel; P. Manivannan; P. Panneerselvam and S. Muig-An (2009). Understanding water deficit stress-induced changes in the basic metabolism of high plants- biotechnologically and sustainably improving agriculture and the ecoenvironment in arid regions of the globe. Critical Rev Biotechnol 29(2):131-151.

Sheikha, S.A. and F.M. AL-Malki (2011). Growth and Chlorophyll Responses of Bean Plants to the Chitosan Applications. European Journal of Scientific Research 50(1):124-134. 
Singh, B. and K. Usha (2003). Salicylic acid induced physiological and biochemical changes in wheat seedlings under water stress. Plant growth regulation 39:137-141.

Somerville, C. and J. Briscoe (2001) Genetic engineering and water. Science 292:2217.

Song, F.B.; D.J. Ying; Z. Lie; H.G. Kun and G. YiQing (1998). Effect of water stress on maize pollen vigour and filament fertility. Acta Agronomica Sinica 24:368-373.

Stibrova, M.; M. Doubravova; A. Brezlova and A. Fridrich (1986). Effects of heavy metals ions on growth and biochemical characteristics of photosynthesis of barley. Photosynthetica 20:416-425.

Tambussi, E.A. and J. Bort (2007). Water use efficiency in $\mathrm{C}_{3}$ cereals under Mediterranean conditions: a review of physiological aspects. Ann Appl Biol 150:307-321.

Tanguilig, V.C.; E.B. Yambao; J.C.O. Tooles and S.K. Dedatta (1987). Water stress effects on leaf elongation, leaf water potential, transpirationand nutrient uptake of rice, maize and soybean. Plant Soil 103:155-168.

Tezara, W.; V.J. Mitchell; S.D. Driscoll and D.W. Lawlor (1999). Water stress inhibits plant photosynthesis by decreasing coupling factor and ATP. Nature 401:914-917

Turner, L.B. (1985). Changes in the phosphorous content of Capsicum annuum $L$ ) leaves during water stress. J Plant Physiology 121:429.

Vurayai, R.; V. Emongor and B. Moseki (2011). Effect of water stress imposed at different growth and development stages on morphological traits and yield of bambara groundnuts (Vigna subterranean L. Verde). American Journal of Plant Physiology 6 (1):17-27.

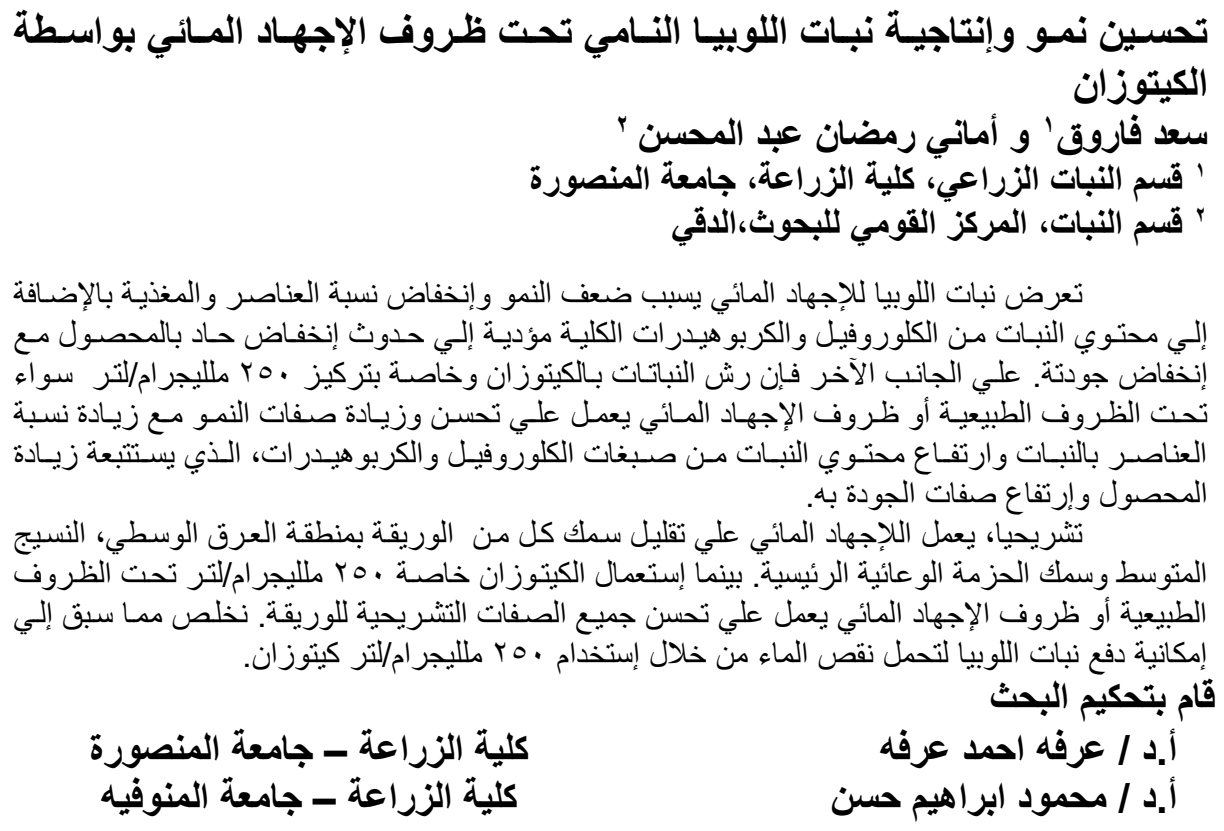


J. Plant Production, Mansoura Univ., Vol. 2 (10), October, 2011 
J. Plant Production, Mansoura Univ., Vol. 2 (10): 1341 - 1358, 2011

Table (5): Effect of water stress and chitosan and their interactions on yield and its quality during the two growing seasons.

\begin{tabular}{|c|c|c|c|c|c|c|c|c|c|c|c|c|c|}
\hline \multicolumn{2}{|c|}{ Treatments } & \multicolumn{2}{|c|}{ Pod yield per plant } & \multicolumn{2}{|c|}{ Nitrogen \% } & \multicolumn{2}{|c|}{ Phosphorous \% } & \multicolumn{2}{|c|}{ Potassium \% } & \multicolumn{2}{|c|}{ Protein \% } & \multicolumn{2}{|c|}{ Carbohydrates \% } \\
\hline $\begin{array}{l}\text { Water } \\
\text { stress } \\
\% \text { FC }\end{array}$ & $\begin{array}{c}\text { Chitosan } \\
(\mathrm{mg} / \mathrm{l})\end{array}$ & $\begin{array}{c}\text { 1st } \\
\text { season }\end{array}$ & $\begin{array}{l}\text { 2nd } \\
\text { season }\end{array}$ & $\begin{array}{c}\text { 1st } \\
\text { season }\end{array}$ & $\begin{array}{l}\text { 2nd } \\
\text { season }\end{array}$ & $\begin{array}{c}\text { 1st } \\
\text { season }\end{array}$ & $\begin{array}{l}\text { 2nd } \\
\text { season }\end{array}$ & $\begin{array}{c}\text { 1st } \\
\text { season }\end{array}$ & $\begin{array}{l}\text { 2nd } \\
\text { season }\end{array}$ & $\begin{array}{c}1 \text { st } \\
\text { season }\end{array}$ & $\begin{array}{l}\text { 2nd } \\
\text { season }\end{array}$ & $\begin{array}{c}\text { 1st } \\
\text { season }\end{array}$ & $\begin{array}{l}\text { 2nd } \\
\text { season }\end{array}$ \\
\hline \multirow{4}{*}{$\begin{array}{l}70 \% \\
F C\end{array}$} & W & \begin{tabular}{|c|}
$16.49 \pm$ \\
$0.746 \mathrm{bcd}$
\end{tabular} & $\begin{array}{l}16.53 \pm \\
0.508 \mathrm{~d}\end{array}$ & \begin{tabular}{c|c|}
$3.87 \pm$ \\
$0.704 \mathrm{bcde}$
\end{tabular} & $\begin{array}{c}3.87 \pm \\
0.704 \mathrm{cde}\end{array}$ & $\begin{array}{c}0.526 \pm \\
0.016 \mathrm{de}\end{array}$ & $\begin{array}{c}0.515 \pm \\
0.023 \mathrm{de} \\
\end{array}$ & $\begin{array}{c}1.26 \pm \\
0.034 \mathrm{cde}\end{array}$ & $\begin{array}{c}1.16 \pm \\
0.210 \mathrm{def}\end{array}$ & $\begin{array}{c}24.20 \pm \\
4.40 \mathrm{bcde}\end{array}$ & $\begin{array}{c}24.20 \pm \\
4.409 \mathrm{cde}\end{array}$ & $\begin{array}{l}38.84 \pm \\
0.298 \mathrm{e}\end{array}$ & $\begin{array}{l}38.28 \pm \\
0.174 d\end{array}$ \\
\hline & 125 & \begin{tabular}{|c|}
$17.75 \pm$ \\
$0.360 \mathrm{bc}$
\end{tabular} & $\begin{array}{c}17.43 \pm \\
0.450 \mathrm{bc}\end{array}$ & $\begin{array}{c}4.13 \pm \\
1.26 \mathrm{abc}\end{array}$ & $\begin{array}{c}4.10 \pm \\
0.080 \mathrm{bc}\end{array}$ & $\begin{array}{l}0.613 \pm \\
0.072 \mathrm{c}\end{array}$ & $\begin{array}{l}0.617 \pm \\
0.070 \mathrm{c}\end{array}$ & $\begin{array}{c}1.36 \pm \\
0.136 \mathrm{bc}\end{array}$ & $\begin{array}{c}1.34 \pm \\
0.200 \mathrm{bc}\end{array}$ & $\begin{array}{c}25.81 \pm \\
7.88 \mathrm{abc}\end{array}$ & $\begin{array}{c}25.66 \pm \\
0.508 \mathrm{bc}\end{array}$ & $\begin{array}{l}41.13 \pm \\
0.833 \mathrm{c}\end{array}$ & $\begin{array}{l}41.65 \pm \\
0.480 \mathrm{~b}\end{array}$ \\
\hline & 250 & \begin{tabular}{|l|}
$22.63 \pm$ \\
$5.381 \mathrm{a}$
\end{tabular} & $\begin{array}{l}19.19 \pm \\
1.806 \mathrm{a}\end{array}$ & $\begin{array}{c}4.50 \pm \\
0.213 \mathrm{a}\end{array}$ & $\begin{array}{c}4.45 \pm \\
0.213 a\end{array}$ & $\begin{array}{l}0.875 \pm \\
0.221 \mathrm{a}\end{array}$ & $\begin{array}{l}0.839 \pm \\
0.120 \mathrm{a}\end{array}$ & $\begin{array}{c}1.55 \pm \\
0.072 \mathrm{a}\end{array}$ & $\begin{array}{c}1.51 \pm \\
0.072 \mathrm{a}\end{array}$ & $\begin{array}{l}28.14 \pm \\
1.33 \mathrm{a}\end{array}$ & $\begin{array}{l}27.85 \pm \\
1.335 \mathrm{a}\end{array}$ & $\begin{array}{l}44.92 \pm \\
2.601 \mathrm{a}\end{array}$ & $\begin{array}{l}43.77 \pm \\
0.703 a\end{array}$ \\
\hline & 500 & \begin{tabular}{|c|}
$16.58 \pm$ \\
$0.560 \mathrm{cde}$
\end{tabular} & $\begin{array}{c}16.63 \pm \\
0.491 \mathrm{~cd}\end{array}$ & $\begin{array}{c}3.75 \pm \\
0.491 \mathrm{bcdef}\end{array}$ & $\begin{array}{c}3.75 \pm \\
0.080 \text { def }\end{array}$ & $\begin{array}{c}0.511 \pm \\
0.007 \mathrm{def}\end{array}$ & $\begin{array}{c}0.508 \pm \\
0.007 \mathrm{de}\end{array}$ & $\begin{array}{c}1.19 \pm \\
0.283 \text { def }\end{array}$ & $\begin{array}{c}1.10 \pm \\
0.023 \text { efg }\end{array}$ & $\begin{array}{c}23.47 \pm \\
3.07 \mathrm{bcdef}\end{array}$ & $\begin{array}{c}23.47 \pm \\
0.508 \mathrm{def}\end{array}$ & $\begin{array}{l}37.86 \pm \\
0.805 f\end{array}$ & $\begin{array}{l}37.34 \pm \\
0.805 \mathrm{e}\end{array}$ \\
\hline \multirow{4}{*}{$\begin{array}{l}50 \% \\
F C\end{array}$} & W & \begin{tabular}{|c|}
$15.26 \pm$ \\
0.336 ef
\end{tabular} & $\begin{array}{l}15.67 \pm \\
0.571 \mathrm{e} \\
\end{array}$ & $\begin{array}{c}3.52 \pm \\
0.080 \text { defg }\end{array}$ & $\begin{array}{c}3.47 \pm \\
0.080 f g h\end{array}$ & $\begin{array}{c}0.472 \pm \\
0.002 \text { efgh }\end{array}$ & \begin{tabular}{|c|}
$0.474 \pm$ \\
0.009 ef \\
\end{tabular} & $\begin{array}{c}1.09 \pm \\
0.183 f g h\end{array}$ & $\begin{array}{c}1.01 \pm \\
0.150 \mathrm{ghi} \\
\end{array}$ & $\begin{array}{c}22.01 \pm \\
0.50 \text { defg }\end{array}$ & $\begin{array}{c}21.72 \pm \\
0.508 \mathrm{fgh}\end{array}$ & $\begin{array}{l}35.32 \pm \\
0.413 \mathrm{~h}\end{array}$ & $\begin{array}{l}34.77 \pm \\
1.207 \mathrm{~g} \\
\end{array}$ \\
\hline & 125 & $\begin{array}{c}16 . \leqslant 9 \pm \\
0.488 \mathrm{cde}\end{array}$ & $\begin{array}{l}16.54 \pm \\
0.570 \mathrm{~d}\end{array}$ & \begin{tabular}{|c|}
$3.92 \pm$ \\
0.84 bcde
\end{tabular} & $\begin{array}{c}3.92 \pm \\
0.84 \mathrm{~cd}\end{array}$ & $\begin{array}{c}0.556 \pm \\
0.030 \mathrm{~cd}\end{array}$ & $\begin{array}{l}0.542 \pm \\
0.009 \mathrm{~d}\end{array}$ & $\begin{array}{c}1.31 \pm \\
0.034 \mathrm{~cd}\end{array}$ & $\begin{array}{c}1.22 \pm \\
0.023 \mathrm{cde}\end{array}$ & $\begin{array}{c}24.39 \pm \\
5.26 \text { bcde }\end{array}$ & $\begin{array}{c}24.49 \pm \\
5.250 \mathrm{~cd}\end{array}$ & $\begin{array}{l}39.70 \pm \\
0.727 \mathrm{~d}\end{array}$ & $\begin{array}{l}38.82 \pm \\
0.480 \mathrm{~d}\end{array}$ \\
\hline & 250 & \begin{tabular}{|c|}
$18.21 \pm$ \\
$0.647 \mathrm{~b}$
\end{tabular} & $\begin{array}{l}17.85 \pm \\
0.960 \mathrm{~b}\end{array}$ & $\begin{array}{c}4.27 \pm \\
0.14 \mathrm{ab}\end{array}$ & $\begin{array}{c}4.24 \pm \\
0.080 \mathrm{ab}\end{array}$ & $\begin{array}{l}0.722 \pm \\
0.127 \mathrm{~b}\end{array}$ & $\begin{array}{l}0.687 \pm \\
0.064 \mathrm{~b} \\
\end{array}$ & $\begin{array}{c}1.45 \pm \\
0.152 \mathrm{ab} \\
\end{array}$ & $\begin{array}{c}1.39 \pm \\
0.128 \mathrm{ab}\end{array}$ & $\begin{array}{l}26.68 \pm \\
0.87 \mathrm{ab}\end{array}$ & $\begin{array}{c}26.53 \pm \\
1.512 \mathrm{ab}\end{array}$ & $\begin{array}{l}42.73 \pm \\
0.238 \mathrm{~b}\end{array}$ & $\begin{array}{l}42.23 \pm \\
0.282 \mathrm{~b}\end{array}$ \\
\hline & 500 & $\begin{array}{l}15.27 \pm \\
0.520 \text { ef }\end{array}$ & $\begin{array}{l}15.54 \pm \\
0.762 \mathrm{e} \\
\end{array}$ & $\begin{array}{c}3.59 \pm \\
0.161 \text { cdef } \\
\end{array}$ & $\begin{array}{c}3.57 \pm \\
0.242 \mathrm{efg} \\
\end{array}$ & $\begin{array}{c}0.499 \pm \\
0.014 \text { defg }\end{array}$ & $\begin{array}{l}0.496 \pm \\
0.011 \mathrm{e} \\
\end{array}$ & $\begin{array}{c}1.14 \pm \\
0.235 \mathrm{efg} \\
\end{array}$ & $\begin{array}{c}1.04 \pm \\
0.100 \mathrm{fgh}\end{array}$ & $\begin{array}{c}22.45 \pm \\
1.01 \mathrm{cdef}\end{array}$ & $\begin{array}{c}22.30 \pm \\
1.820 \mathrm{efg} \\
\end{array}$ & $\begin{array}{l}36.53 \pm \\
0.504 \mathrm{~g} \\
\end{array}$ & $\begin{array}{l}35.89 \pm \\
0.718 \mathrm{f}\end{array}$ \\
\hline \multirow{4}{*}{$\begin{array}{l}30 \% \\
\text { FC }\end{array}$} & W & $\begin{array}{l}13.21 \pm \\
0.935 \mathrm{~g} \\
\end{array}$ & $\begin{array}{l}12.89 \pm \\
0.243 \mathrm{~g} \\
\end{array}$ & $\begin{array}{c}2.98 \pm \\
0.291 \mathrm{~g} \\
\end{array}$ & $\begin{array}{l}2.98 \pm \\
0.291 \mathrm{i} \\
\end{array}$ & $\begin{array}{l}0.422 \pm \\
0.034 \mathrm{~h} \\
\end{array}$ & $\begin{array}{l}0.411 \pm \\
0.021 \mathrm{~g} \\
\end{array}$ & $\begin{array}{l}0.923 \pm \\
0.152 \mathrm{i} \\
\end{array}$ & $\begin{array}{c}0.84 \pm \\
0.201 \mathrm{j} \\
\end{array}$ & $\begin{array}{l}18.66 \pm \\
1.82 \mathrm{~g} \\
\end{array}$ & $\begin{array}{l}18.66 \pm \\
0.496 \mathrm{i} \\
\end{array}$ & $\begin{array}{l}30.00 \pm \\
0.511 \mathrm{k} \\
\end{array}$ & $\begin{array}{l}28.67 \pm \\
0.470 \mathrm{j} \\
\end{array}$ \\
\hline & 125 & \begin{tabular}{|c|}
$15.64 \pm$ \\
0.336 def
\end{tabular} & $\begin{array}{l}15.37 \pm \\
1.694 \mathrm{e}\end{array}$ & $\begin{array}{c}3.38 \pm \\
1.122 \mathrm{efg}\end{array}$ & $\begin{array}{c}3.38 \pm \\
0.080 \mathrm{gh}\end{array}$ & $\begin{array}{c}0.447 \pm \\
0.000 \mathrm{fgh}\end{array}$ & $\begin{array}{c}0.445 \pm \\
0.026 \mathrm{fg}\end{array}$ & $\begin{array}{c}1.04 \pm \\
0.100 \mathrm{ghi}\end{array}$ & $\begin{array}{c}0.94 \pm \\
0.251 \text { hij }\end{array}$ & $\begin{array}{c}21.14 \pm \\
7.01 \mathrm{efg}\end{array}$ & $\begin{array}{l}21.14 \pm \\
0.496 \mathrm{gh}\end{array}$ & $\begin{array}{l}33.22 \pm \\
0.521 \mathrm{i}\end{array}$ & $\begin{array}{l}32.46 \pm \\
1.156 \mathrm{~h}\end{array}$ \\
\hline & 250 & $\begin{array}{l}17.32 \pm \\
0.22 \mathrm{bc} \\
\end{array}$ & $\begin{array}{c}17.09 \pm \\
0.150 \mathrm{bcd}\end{array}$ & $\begin{array}{c}4.03 \pm \\
0.080 \mathrm{abcd}\end{array}$ & $\begin{array}{c}4.03 \pm \\
0.080 \mathrm{bcd}\end{array}$ & $\begin{array}{l}0.601 \pm \\
0.059 \mathrm{c} \\
\end{array}$ & $\begin{array}{l}0.586 \pm \\
0.060 \mathrm{c} \\
\end{array}$ & $\begin{array}{c}1.32 \pm \\
0.152 \mathrm{bcd}\end{array}$ & $\begin{array}{c}1.27 \pm \\
0.057 \mathrm{bcd}\end{array}$ & $\begin{array}{c}25.22 \pm \\
0.50 \mathrm{abcd}\end{array}$ & $\begin{array}{c}25.22 \pm \\
0.508 \mathrm{bcd} \\
\end{array}$ & $\begin{array}{l}40.67 \pm \\
0.282 \mathrm{c} \\
\end{array}$ & $\begin{array}{l}40.51 \pm \\
0.306 \mathrm{c} \\
\end{array}$ \\
\hline & 500 & $14.33 \pm$ & $14.32 \pm$ & $3.26 \pm$ & $3.24 \pm$ & $0.429 \pm$ & $0.430 \pm$ & $0.97 \pm$ & $0.89 \pm$ & $20.41 \pm$ & $20.26 \pm$ & $31.52 \pm$ & $30.92 \pm$ \\
\hline
\end{tabular}




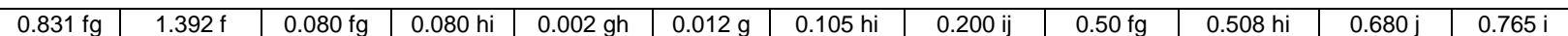

Values are given as mean \pm SD of three replicate. Means in columns by different letters are significantly different at $P<0.05$ by (Duncan's Multiple Range Test) 